\title{
Identification of a New Source of Interference Leached from Polypropylene Tubes in Mass-Selective Analysis
}

\author{
Yuan-Qing Xia, Shefali Patel, Ray Bakhtiar, Ronald B. Franklin, \\ and George A. Doss \\ Department of Drug Metabolism, Merck Research Laboratories, Rahway, New Jersey, USA
}

An interference leached from polypropylene tubes was identified to be a sulfoxide oxidative product of didodecyl 3,3'-thiodipropionate (DDTDP) that is used to prevent oxidative degradation of synthetic polymers. A sulfone oxidative product of DDTDP leached from the polypropylene tubes was also observed. The interfering compounds were isolated by LC and characterized using time-of-flight mass spectrometry and NMR. Authentic sulfoxide and sulfone products of DDTDP were also prepared by reacting DDTDP with hydrogen peroxide reaching an unequivocal structural assignment. In conclusion, when analytes of interest are solubilized in predominantly organic solvents and kept in polypropylene containers, the possibility of contamination from leached chemicals should be taken into account. (J Am Soc Mass Spectrom 2005, 16, 417-421) @ 2004 American Society for Mass Spectrometry

$\mathrm{D}$ ue to its inherent selectivity and specificity, liquid chromatography-mass spectrometry (LCMS) has been used successfully for the qualitative and quantitative determination of drug candidates in biological fluids. However, the presence of low- to mid-range molecular weight ions in LC-MS can lead to interference with the analyte(s) of interest [1-4]. We report the discovery of a new source of interference that was leached from polypropylene tubes by organic solvents. Liquid chromatography coupled with hybrid orthogonal time-of-flight mass spectrometry (LC-TOFMS), ${ }^{1} \mathrm{H}$, and ${ }^{13} \mathrm{C}$ NMR were utilized for identification of the unknown interfering compounds.

\section{Experimental}

\section{Materials and Reagents}

Didodecyl 3,3'-thiodipropionate (DDTDP, (I) was obtained from Sigma Aldrich (St. Louis, MO). Its oxidative products, sulfoxide (II), and corresponding sulfone (III), were synthesized by the addition of $10 \mu \mathrm{L}$ of hydrogen peroxide $(30 \%)$ to $0.2 \mathrm{~mL}$ of (I) $(0.1 \mathrm{mg} / \mathrm{mL})$ prepared in acetonitrile/water $(50 / 50, \mathrm{vol} / \mathrm{vol})$ containing $0.1 \%$ formic acid and kept at room temperature for $30 \mathrm{~min}$. Chemical structures of these compounds are shown in Figure 1. Hydrogen peroxide (30\% purity, ACS grade) was purchased from Fisher Scientific (Fair Lawn, NJ).

Published online February 2, 2005

Address reprint requests to Dr. Y.-Q. Xia, Department of Drug Metabolism, Merck Research Laboratories, Mail Stop RY80L-109, P.O. Box 2000, Rahway, NJ 07065, USA. E-mail: yuanqing_xia@merck.com
Acetonitrile, methanol, hexane, methyl $t$-butyl ether, and ethyl acetate (HPLC grade) were obtained from J. T. Baker (Phillipsburg, PA). Dimethyl sulfoxide (ACS-grade), poly-D, L-alanine, and leucine enkephalin were purchased from Sigma Aldrich (St. Louis, MO). Deuterochloroform was obtained from Aldrich (Milwaukee, WI). All solvents were used without further purification. Nunc 50-mL graduated polypropylene tubes and Wheaton Cryule externally-threaded cryogenic vials $(2-\mathrm{mL})$ were purchased from Fisher Scientific (Pittsburgh, PA).

\section{Generation of the Putative Interfering Compounds from Polypropylene Tubes}

Acetonitrile $(1 \mathrm{~mL})$ was added to each of the 5 polypropylene tubes and kept at room temperature for approximately $10 \mathrm{~min}$. Subsequently, the acetonitrile from all 5 tubes was transferred to a single clean glass tube $(16 \times$ 100 mm, Fisher Brand from Fisher Scientific) and evaporated to dryness under a stream of dry nitrogen gas at ambient temperature. The residue was dissolved in 0.2 $\mathrm{mL}$ of acetonitrile/water $(50 / 50, \mathrm{vol} / \mathrm{vol})$ containing $0.1 \%$ formic acid, pending LC-TOF-MS analysis. Similarly, $1 \mathrm{~mL}$ volumes of other solvents, such as methanol, hexane, methyl $t$-butyl ether, ethyl acetate, and dimethyl sulfoxide were treated as described above.

\section{LC-TOF-MS}

A Micromass Q-TOF-2 (Manchester, UK) equipped with an orthogonal dual-sprayer (a mass and a lock 


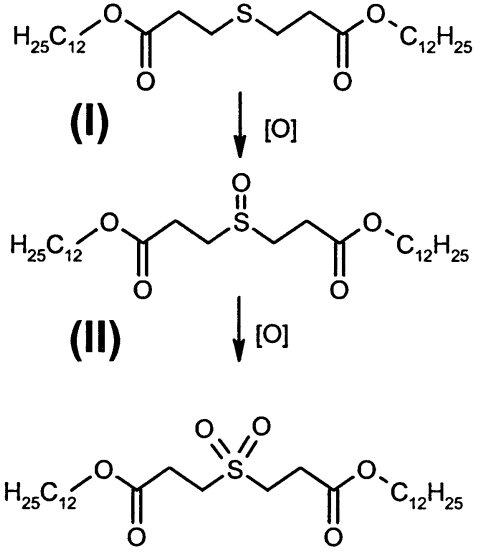

(III)

Figure 1. Oxidation of didodecyl 3,3'-thiodipropionate (I, DDTDP) to the sulfoxide (II) and sulfone (III).

mass sprayer) electrospray source operated in the positive ion mode was used. The capillary and cone voltages were set to $3.0 \mathrm{kV}$ and $40 \mathrm{~V}$, respectively. The source and desolvation temperatures were set to $100{ }^{\circ} \mathrm{C}$ and $350{ }^{\circ} \mathrm{C}$, respectively. The instrument was calibrated in positive ionization mode spanning a range of $\mathrm{m} / \mathrm{z}$ 100-1000 using an aqueous solution of $30 \mu \mathrm{g} / \mathrm{mL}$ poly-D, L-alanine prepared in acetonitrile/water (50/ 50 , vol/vol) containing $0.2 \%$ formic acid. An acquisition rate of one spectrum per second was used for all data collection. Leucine enkephalin, $0.5 \mu \mathrm{g} / \mathrm{mL}$, in acetonitrile/water $(50 / 50, \mathrm{vol} / \mathrm{vol})$ containing $0.1 \%$ formic acid, was used as the lock mass standard that was infused into the source at a flow rate of $15 \mu \mathrm{L} / \mathrm{min}$ with a spray frequency of $5 \mathrm{~s}$. Data were acquired in the continuum mode and the reference and analyte peaks in the mass spectrum were centered using the top $80 \%$ of the peak. For TOF tandem mass spectrometry (TOFMS/MS) experiments, precursor ions at $\mathrm{m} / \mathrm{z} 531$ and 547 were selected and fragmented, using a collision energy of $20 \mathrm{eV}$. The lock mass used for the MS/MS experiment was that of the corresponding precursor ion. MassLynx 4.0 global mass-informatics was used for TOF data acquisition and processing.

The LC system consisted of an Agilent series 1100 system (Piscataway, NJ). A Synergy-Fusion-RP analytical column $(4.6 \times 250 \mathrm{~mm}$ i.d., $4 \mu \mathrm{m}$ particle size; Phenomenex, Torrance, CA) was used for chromatographic separation with an elution gradient consisting

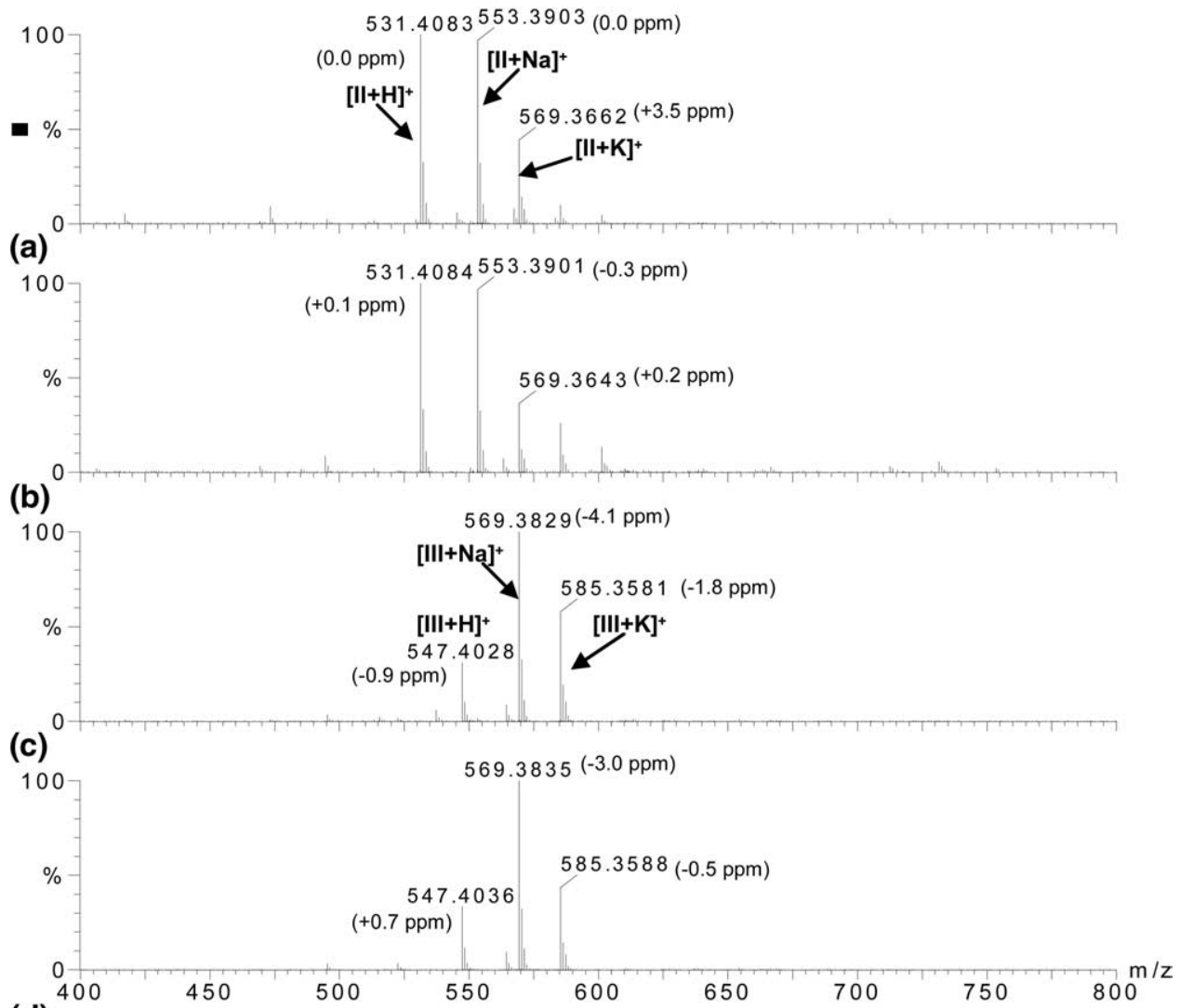

(d)

Figure 2. LC-TOF mass spectra of interfering compound (II) isolated from (a) polypropylene tubes; (b) the reaction of (I) with hydrogen peroxide; those of (III) isolated from (c) polypropylene tubes; (d) the reaction of (I) with hydrogen peroxide. The accurate mass measurement was determined by comparison of measured mass with the theoretical mass and expressed in deviation of part per million (ppm). 
of water and acetonitrile as mobile phases A and B, respectively. Both mobile phases contained $0.1 \%$ formic acid. The gradient was initiated at $50 \% \mathrm{~B}$, followed by a linear increase to $90 \% \mathrm{~B}$ in $45 \mathrm{~min}$ and then maintained at $90 \% \mathrm{~B}$ for $15 \mathrm{~min}$. The flow rate was $1 \mathrm{~mL} / \mathrm{min}$ and column temperature was maintained at ambient conditions. One-fourth of the eluent was directed into the mass spectrometer ion source.

\section{NMR Spectroscopy}

NMR spectra were recorded using a Varian Inova 600 $\mathrm{MHz}$ instrument (Varian, Inc Palo Alto, CA) with a 3-mm inverse detection probe. The sample isolated from the polypropylene tubes was dissolved in deuterochloroform. The deuterium signal from the solvent was used for locking and referencing relative to tetramethyl silane.

\section{Results and Discussion}

During routine LC-MS for quantification of Merck proprietary compounds, we not only observed protonated molecular ions for these compounds, but also intense signals at $m / z 531,547,553,569$, and 585. The signal at $m / z 531$ was particularly troublesome, since it was one mass unit less than the analyte of interest. After carefully examining possibilities, we found that the interference was derived from a 50-mL polypropylene tube used for storage of the internal standard working solution prepared in acetonitrile/water (90/10, vol/ vol). Subsequently, we tested polypropylene tubes from several vendors using acetonitrile, methanol, hexane, methyl $t$-butyl ether, dimethyl sulfoxide, and ethyl acetate as storage solvents. It was determined that solvents containing $>50 \%(\mathrm{vol} / \mathrm{vol})$ of any of the mentioned organic solvents resulted in a similar finding (data not shown). The interfering ions were not detected when the same solvents were added to glass vials. The interfering compounds from the polypropylene tubes were isolated by LC fraction-collection, followed by LC-TOF-MS and NMR.

LC-TOF-MS data of one of the interfering compound (II), which eluted at a retention time of $46.3 \mathrm{~min}$ (data not shown), showed signals corresponding to the protonated molecular ion $(\mathrm{m} / \mathrm{z} 531.4083)$, sodium adduct $(\mathrm{m} / \mathrm{z}$ 553.3903), and potassium adduct $(\mathrm{m} / \mathrm{z}$ 569.3662) (see ${ }^{\circ}$ Figure $2 \mathrm{a}$ ). ${ }^{\circ}$ The ${ }^{\circ}$ accuracy ${ }^{\circ}{ }^{\circ}{ }^{\circ}$ mass ${ }^{\circ}$ measurement ${ }^{\circ}$ was better than $3.5 \mathrm{ppm}$. A TOF-MS/MS spectrum of the precursor $^{\circ}$ ion $^{\circ}$ at $^{\circ} \mathrm{m} / \mathrm{z} 531^{\circ}$ is $^{\circ}$ shown $^{\circ}$ in $^{\circ}$ Figure $^{\circ} 3 a .^{\circ}$ The proposed MS/MS fragmentation of (II) and accurate mass ${ }^{\circ}$ measurement ${ }^{\circ}$ are $^{\circ}$ shown $^{\circ}$ in $^{\circ}$ Figure $^{\circ} 3 a^{\circ}$ and ${ }^{\circ} b .^{\circ}$ The mass accuracy for these product ions was better than 2 ppm except for the product ions at $m / z 123$ and $m / z 105$. We compared the retention time and MS/MS fragmentation of this isolated impurity with those of a standard
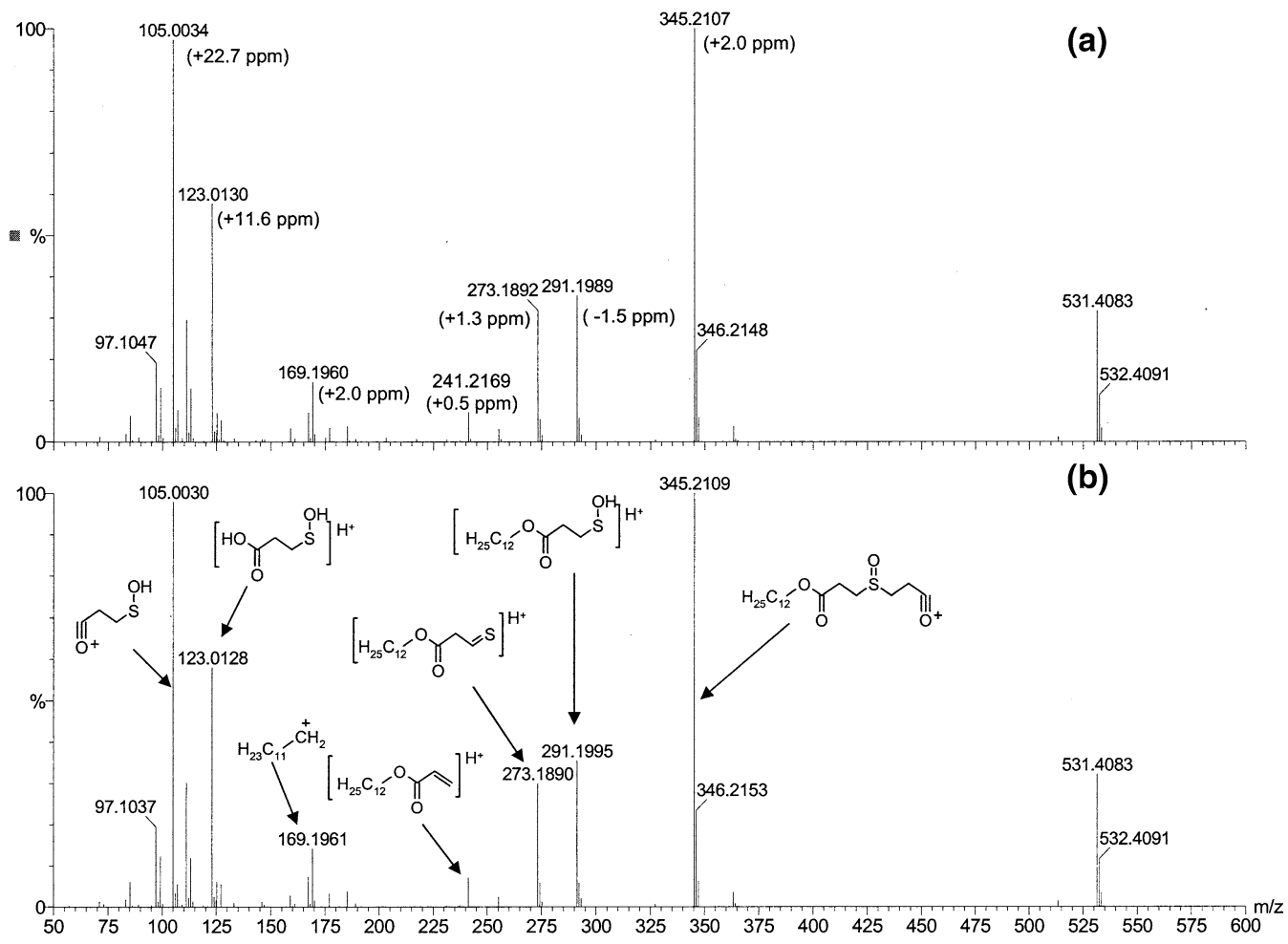

Figure 3. TOF-MS/MS spectra of precursor ion of $\mathrm{m} / \mathrm{z} 531$ obtained (a) from polypropylene tubes; (b) from the reaction of $(\mathbf{I})$ with hydrogen peroxide. The accurate mass measurement was determined by comparison of measured mass with the theoretical mass and expressed in deviation of part per million (ppm). The precursor at $\mathrm{m} / \mathrm{z} 531.4083$ was used as the lock mass. 
<smiles>[10BH2]OC(=O)CCc1cc(C(C)(C)C)c(O)c(C(C)(C)C)c1</smiles>

(IV)

Scheme 1

sample of Irganox 1076 (IV), a commonly used antioxidant ${ }^{\circ}{ }^{\circ}{ }^{\circ}$ synthetic ${ }^{\circ}$ polymers ${ }^{\circ}\left[5,{ }^{\circ} 6\right]^{\circ} .{ }^{\circ} t^{\circ}$ was $^{\circ}$ found ${ }^{\circ}$ that ${ }^{\circ}$ the retention time and MS/MS spectrum of (IV) were not reconcilable with those isolated from the polypropylene tubes, even though they each produced the same protonated molecular ion at $\mathrm{m} / \mathrm{z} 531$.

The structure of (II) was determined from 1D and 2D

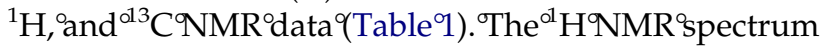
(Figure $\left.^{\circ} 4 \mathrm{a}\right)^{\circ}$ showed $^{\circ}$ characteristic $^{\circ}$ signals $^{\circ}{ }^{\circ}{ }^{\circ}{ }^{\circ}$ long $^{\circ}$ chain esters, e.g., methyl triplet at $0.88 \mathrm{ppm}$ and $\mathrm{COOCH}_{2}$ triplet at $4.12 \mathrm{ppm}$. The NMR signal integrals, combined with the accurate mass data of the protonated molecular ion $(\mathrm{m} / \mathrm{z} 531.4083)$, suggested a symmetrical diester with two $\mathrm{C} 12$ alcohol chains. The acid portion of the ester showed two characteristic $\mathrm{CH}_{2}-\mathrm{CH}_{2}$ signals near 2.8-3.1 ppm. One of the $\mathrm{CH}_{2}$ groups showed distinct nonequivalence indicating the presence of chirality in the molecule. A relative intensity of $\mathrm{M}+2$ peak of the molecular ion at $11 \%$ confirmed the presence of a single $S$ with empirical formula of $\mathrm{C}_{30} \mathrm{H}_{59} \mathrm{O}_{5} \mathrm{~S}$ instead of that at $6.7 \%$ for $\mathrm{C}_{30} \mathrm{H}_{59} \mathrm{O}_{70}$ (Figure 2). This 9 ed to the ${ }^{2}$ proposal of a sulfoxide group connecting two dodecyl propionate ester moieties. In addition, the ${ }^{13} \mathrm{C}$ chemical shifts determined by a $2 \mathrm{D}{ }^{1} \mathrm{H}^{-13} \mathrm{C}$ correlation experiment (Figure $\left.{ }^{\circ} 4 \mathrm{~b}\right)^{\circ}$ further ${ }^{\circ}$ supported ${ }^{\circ}$ the ${ }^{\circ}$ proposed ${ }^{\circ}$ structure.

Antioxidants are widely used to inhibit and retard oxidative degradation of synthetic polymers. Sulfurcontaining compounds are the most important classes of peroxide decomposers, in which one antioxidant molecule destroys several hydroperoxides through the intermediacy ${ }^{\circ}$ of $^{\circ} \mathrm{a}^{\circ}$ sulfur $^{\circ}$ acid $^{\circ}[5] .^{\circ} \mathrm{A}^{\circ}$ search $^{\circ}$ for ${ }^{\circ}$ the

Table 1. Proton and Carbon-13 NMR data for the sulfoxide (II) isolated from polypropulene tubes ${ }^{\mathrm{a}}$

\begin{tabular}{|c|c|c|c|}
\hline Position $^{b}$ & Carbon-13 & & Proton \\
\hline a & 65.8 & 4.12 & $4 \mathrm{H} \mathrm{t} \mathrm{J}=6.9 \mathrm{~Hz}$ \\
\hline b & 28.8 & 1.63 & $4 \mathrm{H}$ quintet $\mathrm{J}=7.1$ \\
\hline c & 26.1 & 1.33 & $4 \mathrm{H} \mathrm{m}$ \\
\hline d & 29.8 & 1.26 & $24 \mathrm{H} \mathrm{m}$ \\
\hline e & 32.2 & 1.25 & $4 \mathrm{H} \mathrm{m}$ \\
\hline f & 23.0 & 1.29 & $4 \mathrm{H} \mathrm{m}$ \\
\hline $\mathrm{g}$ & 14.4 & 0.88 & $6 \mathrm{H} \mathrm{t} \mathrm{J}=7.1$ \\
\hline $\mathrm{h}$ & 27.4 & 2.84 & $4 \mathrm{H} \mathrm{m}$ \\
\hline $\mathrm{i}$ & 27.4 & 2.84 & $4 \mathrm{H} \mathrm{m}$ \\
\hline \multirow[t]{2}{*}{ j } & 47.5 & 2.93 & $2 \mathrm{H} \mathrm{dt} \mathrm{J}=13.2,6.7$ \\
\hline & & 3.07 & $2 \mathrm{H} \mathrm{dt} \mathrm{J}=13.2,7.8$ \\
\hline$q$ & 171.5 & & \\
\hline
\end{tabular}

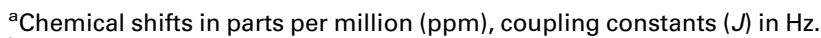
bThe assignment designations are shown in Figure 4.
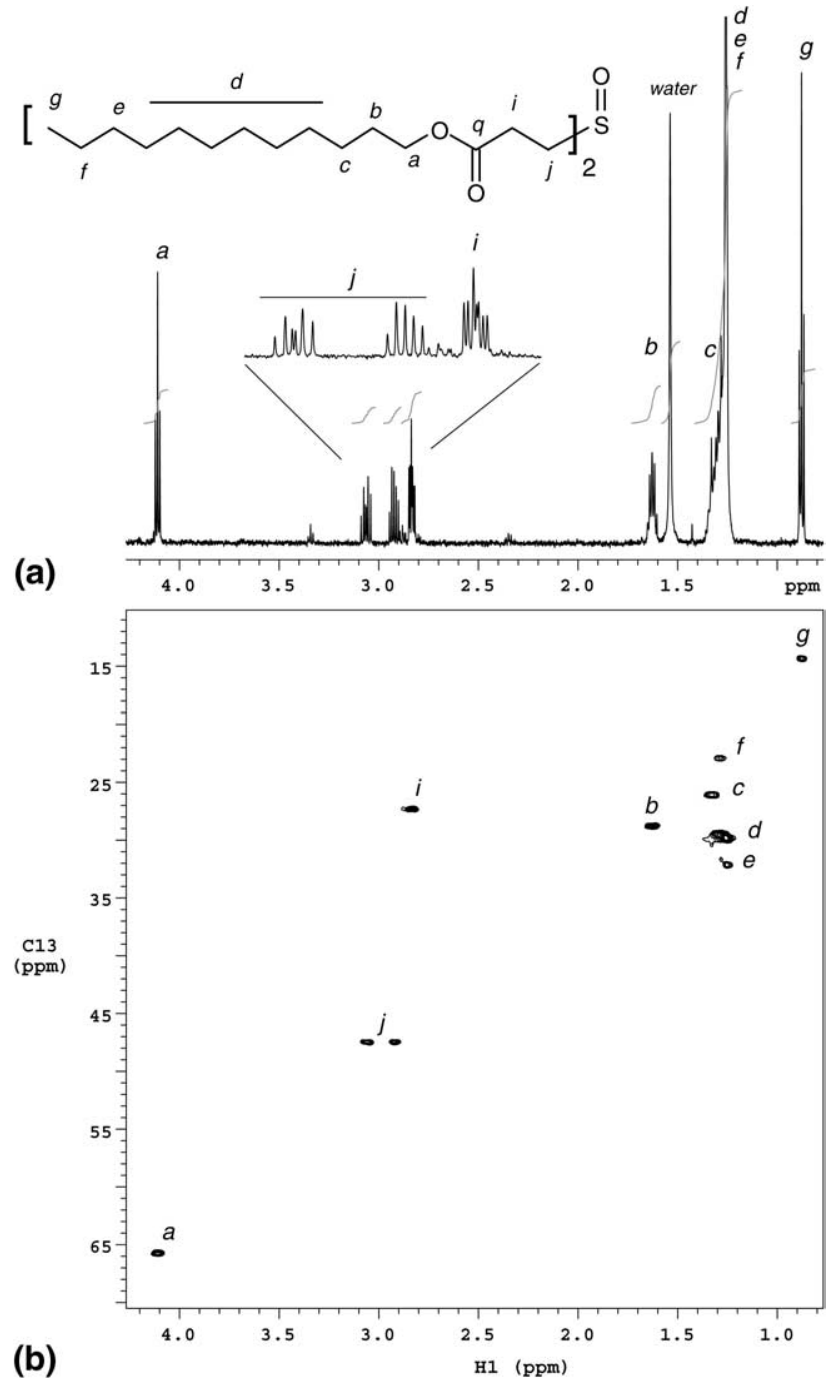

Figure 4. (a) ${ }^{1} \mathrm{H}$ NMR spectrum $(600 \mathrm{MHz})$ of sulfoxide (II); (b) $2 \mathrm{D}{ }^{1} \mathrm{H}-{ }^{13} \mathrm{C}$ one-bond correlation (HSQC) NMR spectrum of sulfoxide (II) isolated from polypropylene tubes.

interfering compound $(\mathrm{m} / \mathrm{z} 531)$ in commercial chemical databases, expecting it to be that of a known additive, was unsuccessful. The corresponding sulfide (I), however, was found to be commercially available, and used widely $^{\circ}$ as $^{\circ}$ an $^{\circ}$ antioxidant ${ }^{\circ}[5] . .^{\circ}$ Hence, ${ }^{\circ}(\mathbf{I})^{\circ}$ was $^{\circ}$ reacted with ${ }^{\circ}$ hydrogen ${ }^{\circ}$ peroxide ${ }^{\circ}$ to $^{\circ}$ produce $^{\circ}(\mathbf{I I})^{\circ}\left(\text { Figure }^{\circ} 1\right)^{\circ}$ for comparison with the interference isolated from polypropylene tubes. The retention time $(46.2 \mathrm{~min})$, TOF-MS $^{\circ}$ spectrum $^{\circ}\left(\right.$ Figure $\left.^{\circ} 2 \mathrm{~b}\right),{ }^{\circ}$ and ${ }^{\circ} \mathrm{TOF}-\mathrm{MS} / \mathrm{MS}^{\circ}$ spectrum $^{\circ}\left(\text { Figure }^{\circ} 3 \mathrm{~b}\right)^{\circ}$ of $^{\circ}(\mathbf{I I})^{\circ}$ matched $^{\circ}$ those ${ }^{\circ}$ of $^{\circ}$ the ${ }^{\circ}$ interference $^{\circ}$ isolated ${ }^{\circ}$ from $^{\circ}$ polypropylene ${ }^{\circ}$ tubes ${ }^{\circ}$ (Figure $2 a^{\circ}$ and Figure ${ }^{\circ} 3$ a). ${ }^{\circ}$ Thus $^{\circ}{ }^{\circ}$ based $^{\circ}{ }^{\circ}{ }^{\circ}$ the ${ }^{\circ}$ LC-TOF-MS ${ }^{\circ}$ and ${ }^{\circ}$ NMR data, the interference compound that had leached from the polypropylenes was determined to be an oxidative product of (I), the sulfoxide (II).

In addition, we found another oxidative product of (I) with a protonated molecular ion at $\mathrm{m} / \mathrm{z} 547$ that leached from the polypropylene tubes. LC-TOF-MS data indicated that this oxidative product of (I) eluted at 
a retention time of $53.4 \mathrm{~min}$ (data not shown) and yielded prominent ions at $m / z 547.4028,569.3829$, and 585.3581 corresponding to the protonated molecular ion, and the sodium and potassium adducts, respectively $^{\circ}\left(\right.$ Figure $\left.^{\circ} 2 \mathrm{c}\right) .^{\circ}$ These $^{\circ}$ data $^{\circ}$ were $^{\circ}$ identical $^{\circ}$ to $^{\circ}$ those observed from the reaction of (I) with hydrogen peroxide'(Figure $2 \mathrm{~d}$ ). ${ }^{\circ}$ Based ${ }^{\circ}$ on ${ }^{\circ}$ he ${ }^{\circ}$ esults ${ }^{\circ}$ of the ${ }^{\circ}$ C-TOF-MS, TOF-MS/MS (data not shown), and authentic standards, we confirm this isolated interfering compound to be a second oxidative product of (I), the sulfone (III).

A combination of TOF-MS, TOF-MS/MS, and NMR data, as well as the availability of authentic compounds, proved to be conclusive in the structural assignment of the sulfoxide and sulfone compounds that were leached from polypropylene tubes containing organic solvents. These compounds were the oxidative sulfoxide (II), $\mathrm{m} / \mathrm{z}$ 531 , and sulfone (III), $m / z 547$, products of the antioxidant compound (I), the sulfide compound of DDTDP. The formation of the oxidative products, (II) and (III), is depicted ${ }^{\circ}{ }^{\circ}$ Figure $^{\circ} 1$.

\section{Conclusions}

A new source of interference that originated from polypropylene tubes was identified to be the sulfoxide $(m / z$ 531) product of an antioxidant, didodecyl 3,3'thiodipropionate (DDTDP), which is used in polymer manufacturing in order to prevent oxidative degradation. Later, another interfering compound $(m / z 547)$ was detected and was determined to be the sulfone product of DDTDP. The interfering ions of $m / z 531$ and 547, and their sodium and potassium adducts at $m / z 553,569$, and 585 may compromise qualitative or quantitative LC-MS bioanalysis if polypropylene tubes are used for storage of analytes in large volumes of organic solvents. The problem of the leaching of impurities is likely to be particularly problematic when using single stage MS with minimal chromatographic separation. Thus, adequate chromatographic separation is highly recommended in order to reduce the possibility of encountering these or other interferences. When available, MS/MS specificity is another attractive alternative to guard against possible interferences. Lastly, glass containers instead of polypropylene containers should be considered for the storage of the analytes dissolved in large volumes of organic solvents.

\section{Acknowledgments}

The authors thank Drs. Kevin Bateman and John M. Ballard of the Merck Research Laboratories for their critical and thoughtful comments, and Professor E. Bryan Coughlin of the University of Massachusetts (Amherst, MA) for the supply of standard Irganox 1076.

\section{References}

1. Verge, K. M.; Agnes, G. R. Plasticizer Contamination from Vacuum System O-Rings in a Quadrupole Ion Trap Mass Spectrometer. J. Am. Soc. Mass Spectrom. 2003, 13, 901-905.

2. Purves, R. W.; Gabryelski, W.; Li, L. The Effect of Using Silicon Based Diffusion Pump Fluid on Spectral Quality in an Electrospray Ionization Ion Trap/Time-of-Flight Mass Spectrometer. Rev. Sci. Instrum. 1997, 68, 3252-3253.

3. Aebi, B.; Henion, J. D. The Reduction of Chemical Noise in an Atmospheric Pressure Ionization/Ionspray Interface for Mass Spectrometry. Rapid Commun. Mass Spectrom. 1996, 10, 947-951.

4. Loyo-Rosales, J. S.; Rosales-Rivera, G. C.; Lynch, A. M.; Rice, C. P.; Torrents, A. Migration of Nonylphenol from Plastic Containers to Water and a Milk Surrogate. J. Agric. Food Chem. 2004, 52, 2016-2020.

5. Al-Malaika, S. Oxidative Degradation and Stabilization of Polymers. Int. Mater. Rev. 2003, 48, 165-185.

6. Hinsken, H.; Moos, S.; Pauquet, J.-R.; Zweifel, H. Degradation of Polyolefins During Melt Processing. Polym. Degrad. Stab. 1991, 34, 279-293. 